\section{Two new Asteridiella species from Tamil Nadu, India}

\author{
V.B. Hosagoudar ${ }^{1}$, K. Ravikumar ${ }^{2}$ \& G.R. Archana ${ }^{3}$ \\ 1,3 Tropical Botanic Garden and Research Institute, Palode, \\ Thiruvananthapuram, Kerala 695562, India \\ ${ }^{2}$ Foundation for Revitalisation of Local Health Traditions (FRLHT), \\ Bangalore, Karnataka 560064, India \\ Email: 1 vbhosagoudar@ rediffmail.com
}

During a survey of endemic and endangered plants in the Shenbaganur reserve forests near Kodaikanal in Tamil Nadu, Symplocos anamallayana Bedd. (Symplocaceae) was found severely infected with a black mildew fungus. The fungus was peeled-off by nail polish technique to study in natural conditions (Hosagoudar \& Kapoor 1984; Hosagoudar 1996). The fungal colonies revealed presence of two new fungal taxa of the genus Asteridiella McAlpine, easily distinguished by oblong and globose head cells of appressoria and presence of three and four septate ascospores.

\section{Asteridiella kodaikanalensis sp. nov.} (Fig. 1)

Coloniae amphigenae, plerumque epiphyllae, densae, ad $2 \mathrm{~mm}$ diam., coloniae epiphyllorum confluentes et coalescentes. Hyphae rectae vel subrectae, oppositae acuteque ramosae, laxe vel dense reticulatae, cellulae 11-32 x 6-10 $\mu \mathrm{m}$. Appressoria alternata, unilateralis, recta, antrorsa, 14-27 $\mu \mathrm{m}$ longa; cellulae basilares cylindraceae vel cuneatae, 3-11 $\mu \mathrm{m}$ longae; cellulae apicales plerumque rectae, globosae, integrae, ovatae, 11-16 x 9-13 $\mu \mathrm{m}$. Phialides appressoriis mixtus, oppositae vel unilateralis, ampulliformes, 16-24 x 6-10 $\mu \mathrm{m}$. Perithecia globosa, aggregata, ad $168 \mu \mathrm{m}$ diam.; cellulae peritheciales conoideae vel mammiformes, ad $32 \mu \mathrm{m}$ longae; ascosporae cylindraceae, 4-septatae, constrictae ad septatae, 35-42 × 16$18 \mu \mathrm{m}$.

Colonies amphigenous, mostly epiphyllous, dense, up to $2 \mathrm{~mm}$ in diameter, confluent and cover an entire upper surface of the leaves. Hyphae straight to substraight, branching

Date of publication (online): 26 August 2009

Date of publication (print): 26 August 2009

ISSN $0974-7907$ (online) | 0974-7893 (print)

Editor: R.K. Verma

\section{Manuscript details:}

Ms \# 02060

Received 13 September 2008

Finally accepted 19 June 2009

Citation: Hosagoudar, V.B., K. Ravikumar \& G.R. Archana (2009). Two new Asteridiella species from Tamil Nadu, India. Journal of Threatened Taxa 1(8): 434-436.

Copyright: ( V.B. Hosagoudar, K. Ravikumar \& G.R. Archana 2009. Creative Commons Attribution 3.0 Unported License. JoTT allows unrestricted use of this article in any medium for non-profit purposes, reproduction and distribution by providing adequate credit to the authors and the source of publication.

Acknowledgement: We thank Dr. S. Ganeshan, Director, TBGRI, Palode for the facilities.

OPEN ACGESS | FREE DOWNLOAD CC (7) ৫ு opposite at acute angles, loosely to closely reticulate, cells 11-32 x 6-10 $\mu \mathrm{m}$. Appressoria alternate, unilateral, straight, antrorse, 14-27 $\mu \mathrm{m}$ long; stalk cells cylindrical to cuneate, $3-11 \mu \mathrm{m}$ long; head cells mostly straight, globose, ovate, entire, 11-16 x 9-13 $\mu \mathrm{m}$. Phialides mixed with appressoria, opposite, unilateral, ampulliform, 16-24 x 6-10 $\mu \mathrm{m}$. Perithecia globose, grouped, up to $168 \mu \mathrm{m}$ in diam.; perithecial wall cells conoid to mammiform, up to $32 \mu \mathrm{m}$ long; ascospores cylindrical, 4-septate, constricted at the septa, 35-42 x 16-18 $\mu \mathrm{m}$.

Etymology: Species named after collection locality

\section{Material examined}

Type: 20.ii.2006, On leaves of Symplocos anamallayana Bedd. (Symplocaceae), Shenbaganur Shola, Kodaikanal, Tamil Nadu, India, coll. K. Ravi Kumar HCIO 46807 (type), TBGT 2148 (isotype) (Myco Bank \# MB5140116).

This species is similar to Asteridiella fidelis (Toro) Hansf., known on Symplocos theiformis from Philippines in which ascospores are four septate (Hansford 1961). However, $A$. kodaikanalensis differs from it in having predominantly globose but entire head cells of appressoria and also phialides produced on the mycelial branch where appressoria are also borne.

\section{Asteridiella shenbaganurensis sp. nov.} (Fig. 2)

Coloniae amphigenae, plerumque epiphyllae, densae, ad $2 \mathrm{~mm}$ diam., confluentes et coalescentes epiphyllorum. Hyphae rectae vel subrectae, oppositae vel alternate acuteque vel laxe ramosae, laxe vel dense reticulatae, cellulae 16-42 x 8-10 $\mu \mathrm{m}$. Appressoria alternata, unilateralis, recta vel saepe curvula, antrorsa vel subantrorsa, 17-42 $\mu \mathrm{m}$ longa; cellulae basilares plerumque unicellularis, raro uniseptatis, cylindraceae vel cuneatae, 4-18 $\mu \mathrm{m}$ longae; cellulae apicales plerumque rectae, saepe curvulae, oblongae, ovatae, clavatae, plerumque integrae, saepe angularis vel raro sublobatae, 9-24 x 8-16 $\mu \mathrm{m}$. Phialides appressoriis mixtus, oppositae, alternatae vel unilateralis, ampulliformes, 20-37 x 8-10 $\mu \mathrm{m}$. Perithecia globosa, aggregata, ad $208 \mu \mathrm{m}$ diam.; cellulae peritheciales conoideae vel mammiformes, ad $32 \mu \mathrm{m}$ longae; ascosporae ellipsoideae vel fusiformes, rectae vel curvulae, 3-septatae, constrictae ad septatae, 43-49 × 14-19 $\mu \mathrm{m}$.

Colonies amphigenous, mostly epiphyllous, dense, up to $2 \mathrm{~mm}$ in diameter, confluent and cover an entire upper surface of the leaves. Hyphae straight to substraight, branching opposite to alternate at acute to wide angles, loosely to closely reticulate, cells 16-42 x 8-10 $\mu \mathrm{m}$. Appressoria alternate, unilateral, straight to often slightly curved, antrorse to subantrorse, 17-42 $\mu \mathrm{m}$ long; stalk cells mostly unicellular, rarely 1-septate, cylindrical to cuneate, 4-18 $\mu \mathrm{m}$ long; head cells mostly straight, often curved, oblong, ovate, clavate, mostly entire, often angular, rarely sublobate, 9-24 x 8-16 $\mu \mathrm{m}$. Phialides mixed with appressoria, opposite, alternate, unilateral, ampulliform, 20-37 x 8-10 $\mu \mathrm{m}$. Perithecia globose, grouped, up to $208 \mu \mathrm{m}$ in diam.; perithecial wall cells conoid to mammiform, up to $32 \mu \mathrm{m}$ long; ascospores ellipsoidal to fusiform, straight to curved, 3-septate, constricted at the septa, 43-49 x 14-19 $\mu \mathrm{m}$. 


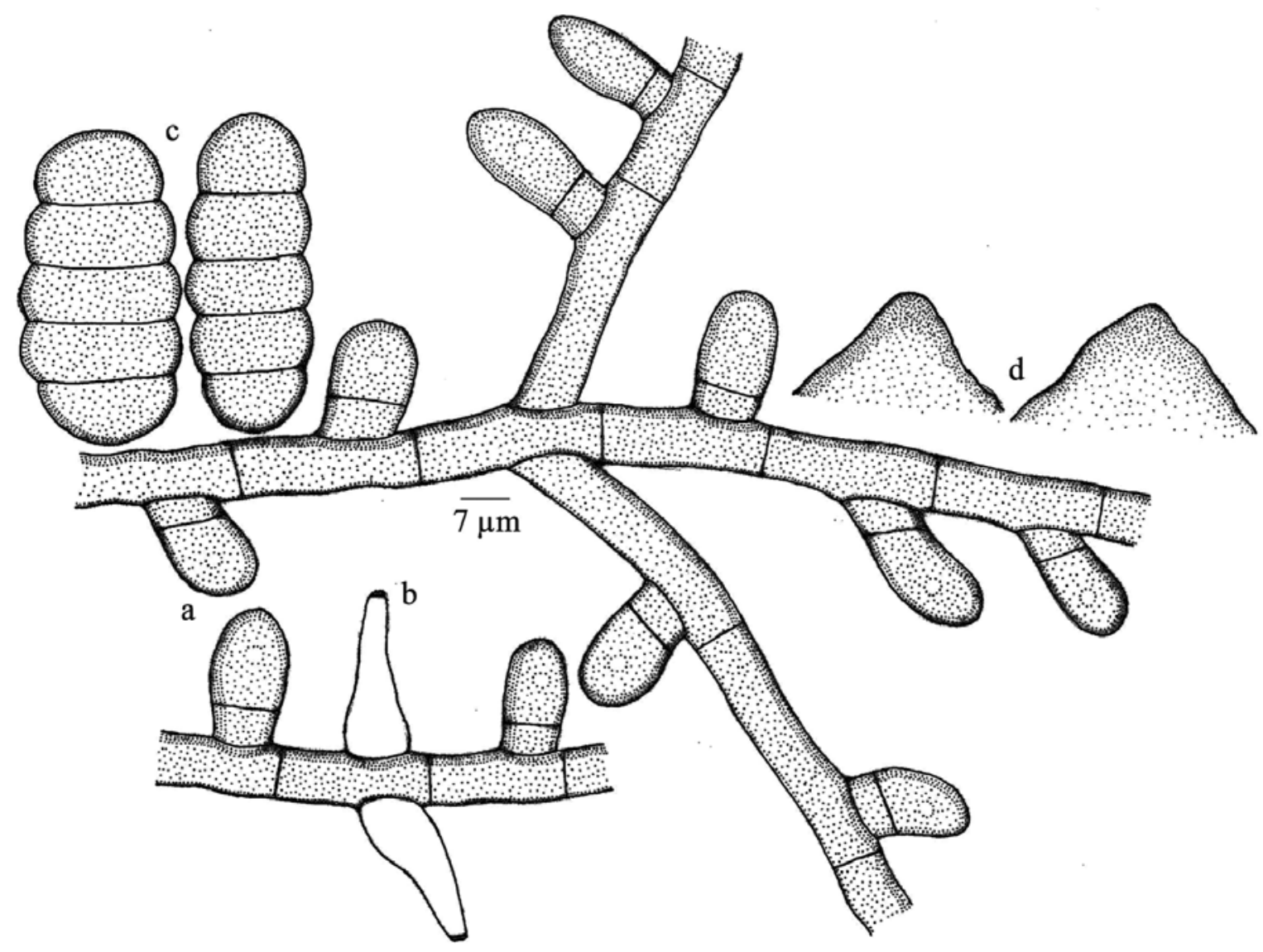

Figure 1. Asteridiella kodaikanalensis sp. nov.

a - Appressorium; b - Phialide; c - Ascospores; $d$ - Perithecial wall cells

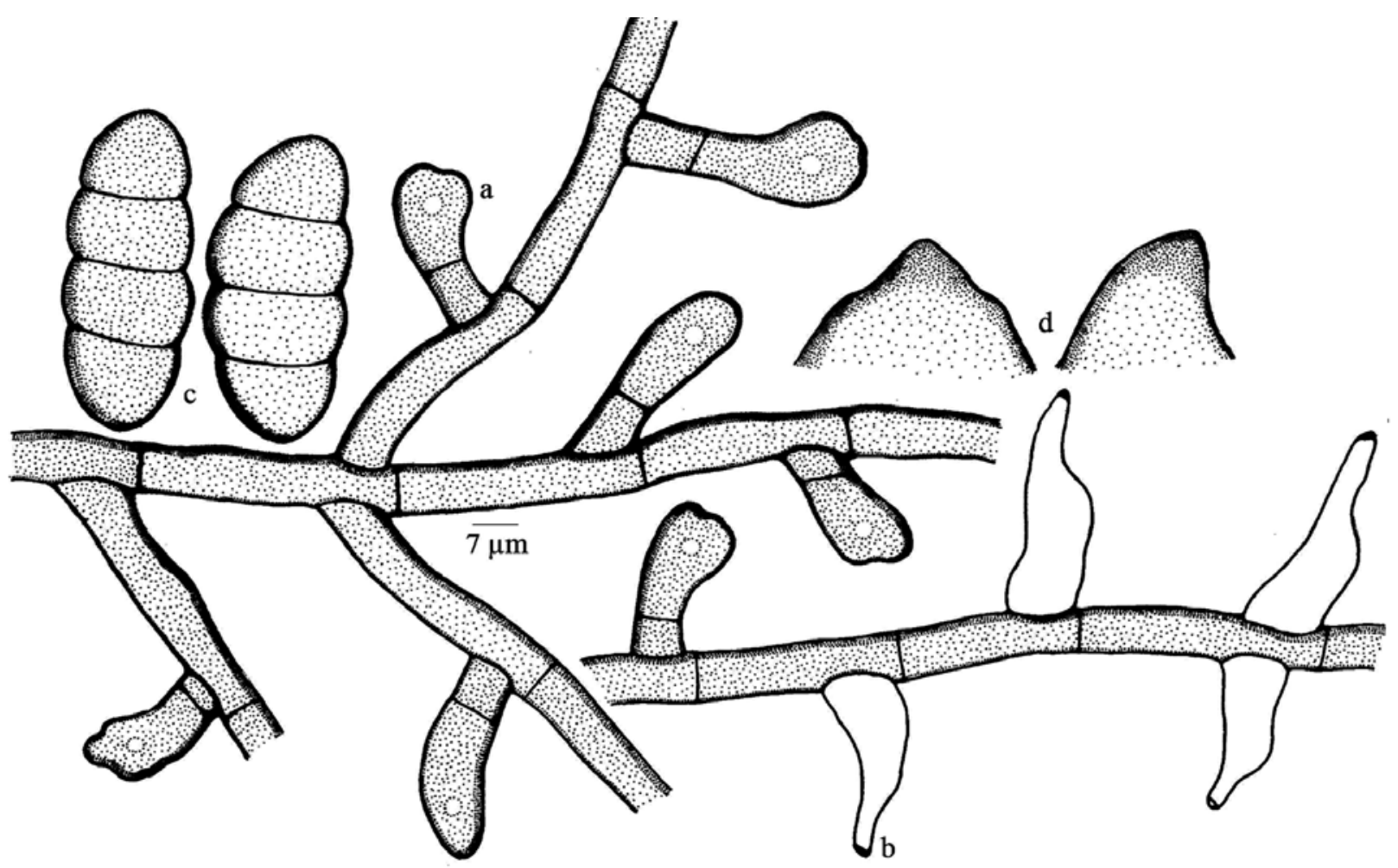

Figure 2. Asteridiella shenbaganurensis sp. nov.

a - Appressorium; b - Phialide; c - Ascospores; d - Perithecial wall cells 
Etymology: Species named after collection locality

\section{Material examined}

Type: 20.ii.2006, On leaves of Symplocos anamallayana Bedd. (Symplocaceae), Shenbaganur Shola, Kodaikanal, Tamil Nadu, India, coll. K. Ravi Kumar HCIO 46808 (type), TBGT 2149 (isotype) (Myco Bank \# MB5140117).

This species is similar to Asteridiella singalensis Hansf., reported on Symplocos sp. from Sri Lanka, in having 3-septate ascospores (Hansford 1961). However, differs from it in having distinctly oblong and entire to angular head cells of appressoria.
Further, phialides are mixed with appressoria and having smaller ascospores.

\section{References}

Hansford, C.G. (1961). The Meliolineae. A Monograph. Sydowia Beih. 2: $1-806$.

Hosagoudar, V.B. (1996). Meliolales of India. BSI, Calcutta, 363pp.

Hosagoudar, V.B. \& J.N. Kapoor (1985). New technique of mounting meliolaceous fungi. Indian Phytopathol. 38: 548-549.

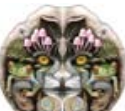

